\title{
Flexible probe for in vivo quantification of corneal epithelium permeability through non- invasive tetrapolar impedance measurements.
}

\author{
A. Guimerà ${ }^{1,2, *}$, X. Illa ${ }^{1,2}$, E. Traver ${ }^{4}$, M. Plata-Cordero ${ }^{3}$, J. Yeste $^{1,2}$, C.Herrero ${ }^{4}$, \\ C.Lagunas ${ }^{4}$, MJ. Maldonado ${ }^{3}$, R. Villa ${ }^{1,2}$ \\ ${ }^{1}$ Institut de Microelectrònica de Barcelona IMB-CNM (CSIC), Bellaterra 08193, \\ Barcelona, Spain \\ ${ }^{2}$ CIBER-BBN, Networking Center on Bioengineering, Biomaterials and Nanomedicine, \\ Zaragoza Spain \\ ${ }^{3}$ IOBA-Eye Institute, University of Valladolid. Valladolid, Spain. \\ ${ }^{4}$ Laboratorios SALVAT S.A., Esplugues de Llobregat, 08950. Barcelona, Spain. \\ *E-mail: anton.guimera@csic.es
}

\begin{abstract}
Studies concerning the functional status of the corneal epithelium are of special interest due to its key role in preventing ocular surface disease and corneal infections. In particular, quantitative measurements of the epithelium permeability translayer electrical resistance (TER) have been proven as a sensitive in vitro test for evaluation of the corneal barrier function. In a recent work from the authors(Guimera et al. 2012), a novel method to non-invasively assess the corneal epithelial permeability by using tetrapolar impedance measurements, based on the same TER theoretical principles, was presented and validated using a rigid sensing device. In this work, the usability of this method has been dramatically improved by using SU-8 photoresist as a substrate material. The flexibility of this novel sensing device makes no need to apply pressure on the cornea to ensure the electrical contact between the electrodes and the corneal surface. The feasibility of this flexible sensor has been evaluated in vivo by increasing the permeability of rabbit corneal epithelium. For that, different concentrations of benzalkonium chloride (BAC) solution were instilled on different rabbit corneas. The obtained results have been compared with measurements of the permeability to sodium fluorescein of different excised corneas, a wellknown method used to evaluate the corneal barrier function, to demonstrate the feasibility of this novel flexible sensor for quantifying the corneal epithelium permeability in vivo in a non-invasive way.
\end{abstract}

Keywords: Electrical impedance spectroscopy; Corneal epithelium; Permeability; Barrier function; Translayer electrical resistance; Non-invasive

\section{Introduction}

The animal cornea is a basic transparent structure containing two covering layers, epithelium and endothelium, and a central collagen stroma. The corneal epithelium is the outer surface of the cornea and serves as a barrier to prevent chemical, biological, and physical insults of the environment from entering the eye. This barrier function is critical for corneal homeostasis, including maintenance of corneal transparency (Kinoshita et al. 2001). Specifically, tight and adherens junctions, which are intercellular junctional structures, are essential for epithelial barrier function and epithelial adhesion (Schulzke et al. 2012). Therefore, any change in the expression and distribution of these junction proteins alters the corneal barrier function (Kimura et al. 2008; Swamynathan et al. 2011).

It is also well-known that disruption of the corneal barrier function can cause ocular irritation and is a risk factor for microbial keratitis. In particular, it has not only been related with the more common infectious and non-infectious inflammatory corneal conditions (Paiva et al. 2006), but also with pathologies caused by contact lens wear (Kimura et al. 2011; Kimura et al. 2008) or by instillation of different ophthalmic topical medications (Ayaki and Iwasawa 2011). In this last case, the insult is produced by the components used as preservatives and surfactants needed to enhance the effect and 
comfort of the topically instilled medication. In particular, to evaluate the cytotoxic effects of different medicines and solutions that require long-term use, such as antiglaucoma drugs, disinfecting and storage solutions for soft contact lenses or artificial tears different studies have been performed (Cavet et al. 2010; Furrer et al. 2002; Lehmann et al. 2010).

Above all, one of the more common preservatives used in the formulation of ophthalmic medications in the present days is benzalkonium chloride (BAC). It is a quaternary ammonium compound whose antimicrobial activity arises from its ability to disrupt cell membranes and potentiate cell death. Besides its antimicrobial activity, BAC is cytotoxic to the corneal tissues by inducing loss of tight junctions between cells, which is directly related with the increase of the epithelium permeability (Baudouin et al. 2010; Swan 1944). Moreover, these changes appear to be both dose- and time-dependent (Chen et al. 2011).

To study the corneal barrier function, quantitative measurements of the epithelium permeability are required. In humans, in vivo fluorophotometric methods are used for this purpose, but the substantial variability between repeated measurements denotes that this single-drop procedure is unreliable for monitoring individual patient changes (Kinoshita et al. 2001; McNamara et al. 1997). In animal experimentation, evaluation of the corneal barrier function can be more accurate by using invasive in-vitro methods. The most common used technique is based on the measurement of the corneal permeability to carboxy fluorescein, but as it has been anticipated, excised corneas from euthanized animals are required. Apart from this method, measurement of the electrical properties of the cornea, such as electrical resistance and trans-corneal potential (Trans-layer Electrical Resistance, TER), has been proven as a sensitive test for detecting variations on the permeability of in-vitro corneal tissues (Ma et al. 2007; Rojanasakul and Robinson 1990). Recently, this approach has been modified in order to be implemented in living animals (Chen et al. 2012; Uematsu et al. 2007). This in vivo TER uses $\mathrm{Ag} / \mathrm{AgCl}$ electrodes inserted into the anterior chamber of the eye through a small incision in the peripheral cornea. Although this technique validates the use of the electrical properties of the cornea to analyze variations on its permeability and demonstrating that exposure to BAC can quickly lead to disruption of the cornea barrier function, there is still no available technique to evaluate quantitatively the corneal permeability in a non-invasive and reliable way which could be used in humans.

In this work, a method based on a flexible probe used to perform non-invasive TER measurements in in vivo conditions is presented. The novel sensing device takes advantage of the previous results reported by the authors (Guimera et al. 2010) where an electrical model based on tetrapolar impedance measurements performed with electrodes placed on the surface of the cornea was presented. Therein, it was concluded that with this method, the different electrical properties for the three primary layers of the cornea (epithelium, stroma and endothelium) could be discerned depending on the sensor geometry or the frequency used. The practical feasibility of the proposed method has been validated in a recent work where measurements were carried out with a rigid sensing device placed on the corneal surface (Guimera et al. 2012). From the obtained results, it was concluded that the method can be used to detect alterations in the epithelial permeability. However, the pressure needed to ensure the electrical contact between the electrodes and the corneal surface limited its performance.

In this work, we present an evolution of that rigid sensing device based on the use of SU-8, a well-known negative photoresist (Keller et al. 2008; Lorenz et al. 1997), in order to fabricate a flexible sensing device. The use of a polymeric substrate for the previously described sensing device will allow us to increase the usability and performance of the proposed method. Moreover, the main weaknesses of the previous rigid probe will be overcome as no pressure is needed for ensuring the electrical contact of the electrodes. 
Finally, the flexible sensing device allows increasing the electrode separation, which results in a sensitivity improvement. To assess and validate the sensitivity of the fabricated sensing device in in vivo conditions, different concentrations of benzalkonium chloride (BAC) solution were instilled on different rabbit corneas. This strategy leads to different increases on the corneal epithelial permeability that enables the discrimination between the different grades of corneal insult when measured with the proposed sensing device. Finally, the impedance measurements performed with the presented sensing device have been compared with measurements of the permeability to sodium fluorescein of different corneas after excising.

\section{Materials and methods}

\section{Impedance sensor}

\section{Sensor design}

As it has been mentioned, the sensor presented here is an evolution of the methodology presented in two previous works (Guimera et al. 2012; Guimera et al. 2010), where the authors showed the feasibility of performing non-invasive TER measurements in in vivo conditions by placing four electrodes on the corneal surface. However, the proposed method showed some drawbacks that should be improved in order to increase its usability and performance. Basically, the sensor device presented in the last of those previous works was based on a rigid substrate, requiring a pressure to ensure electrical contact between the electrodes and the corneal surface and limiting the maximum separation between the electrodes.

The measurement method is based on tetrapolar impedance measurements. When compared to the most common bipolar method, the tetrapolar method offers the advantage of minimizing, and ideally cancelling, the parasitic effects of the electrodeelectrolyte interface impedances (Schwan and Ferris 1968). In a tetrapolar system, the electrical properties of each sub-volume of the tissue have a different contribution to the measured impedance. Then, it is intuitively understood that the volumes close to the electrodes contribute more than volumes far away from the electrodes (Geselowitz 1971; Grimnes and Martinsen 2007). Therefore, the correct choice of the electrode size and position is important for focusing the measurement on the region of interest. Following those guidelines, the contribution of each cornea layer to the measured impedance has been analyzed by using a finite element method (FEM) tool as previously reported (Guimera et al. 2010; Yang and Patterson 2008). Because of the direct influence of the sensor width $\left(\mathrm{W}_{\mathrm{S}}\right)$ to the spatial resolution of the measurement, different geometrical relationships between electrodes were designed (sketch shown in Fig. 1a). In Fig. 1b, the contribution of each corneal layer to the total measured impedance for four different sensors width is shown. It is worthy to note that the contribution of each layer depends on the frequency, being in the low frequency range (frequencies below $50 \mathrm{kHz}$ ) where the corneal epithelium has the largest contribution. However, at this frequency range the contribution of the tear film is also important. As has been previously observed (Guimera et al. 2012), little variations in the tear film thickness produce variations on the performed measurements. Consequently, those four different electrode configurations will be evaluated in experimental conditions in order to discern which works better to evaluate the epithelium permeability. Accordingly, the final sensing device, which is shown in Fig. $1 \mathrm{a}$, consists of 10 electrodes that allow us to implement the 4 different electrode configurations in function of the electrodes selected to perform the measurement. 

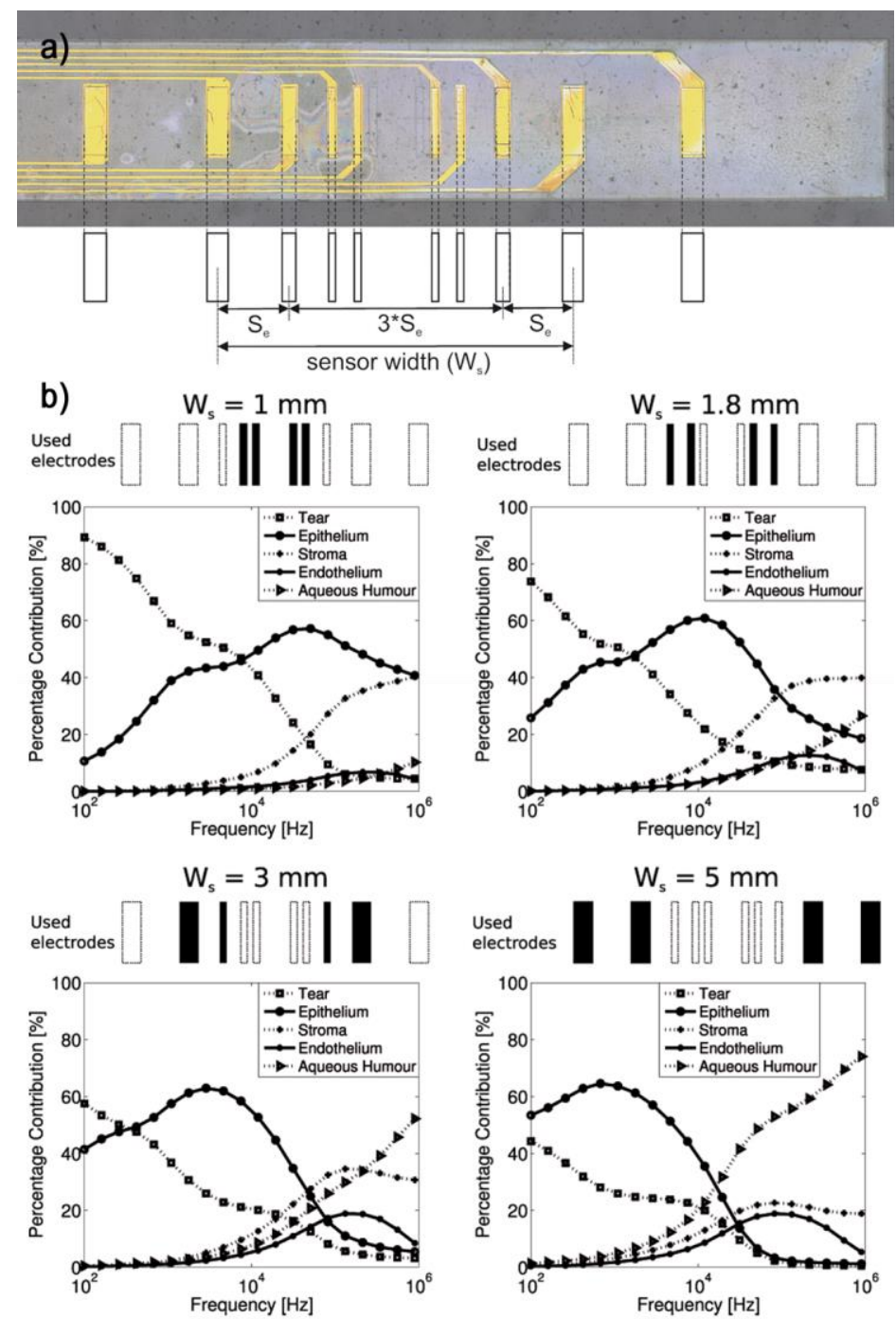

Fig. 1 (a) Image of the SU-8 sensing device composed of ten gold electrodes and schematic representation of the geometrical relationship between electrodes. (b) For the four different electrode geometries $\left(\mathrm{W}_{\mathrm{s}}=1,1.8,3\right.$ and $5 \mathrm{~mm}$ ): (up) schematic representation of the electrode configuration (with the used electrodes filled in black). (down) Contribution of each cornea layer to the total measured impedance as function of frequency obtained by the FEM tool

\section{Sensor fabrication}

The fabrication process of the flexible SU-8 sensing device starts with a thermal oxidation of a 4-inch silicon wafer. $400 \mathrm{~nm}$ of $\mathrm{SiO}_{2}$ were grown to serve as a sacrificial layer for the final release of the devices. Then, the wafer was heated up to $200^{\circ} \mathrm{C}$ in a hotplate in order to enhance the adhesion of the silicon oxide to the SU-8 negative photoresist (2025, Microchem, USA) which was poured over the wafer. To obtain a 25 $\mu \mathrm{m}$ thick structural layer, the wafer was spin coated and baked at $65^{\circ} \mathrm{C}$ for $3 \mathrm{~min}$ and at $95^{\circ} \mathrm{C}$ for $20 \mathrm{~min}$. UV-exposure was made through the mask where the device structure is defined using a dose of $140 \mathrm{~mJ} / \mathrm{cm}^{2}$. After the exposure, the wafer was heated up to $65^{\circ} \mathrm{C}$ for $1 \mathrm{~min}$, ramped up to $95^{\circ} \mathrm{C}$, baked at this temperature for 8 min and cooled down on the hotplate to ambient temperature. Afterwards, the wafer was developed in a propylene glycol methyl ether acetate (PGMEA)-based (mr Dev-600) bath for $3 \mathrm{~min}$, rinsed with isopropyl alcohol (IPA) and dried with a $\mathrm{N}_{2}$ gun. A hard bake at $120^{\circ} \mathrm{C}$ for $20 \mathrm{~min}$ in a hotplate was also applied to the wafer in order to finalize the photolithographic process. 
Evaporation of $20 \mathrm{~nm}$ of titanium (that acts as an adhesion layer) and $200 \mathrm{~nm}$ of gold were subsequently performed using an electron beam gun in a Univex 450B coating system (Oerlikon Leybold, Germany). However, before evaporation, a low-power oxygen plasma etching was applied to the wafer to improve metal adhesion to the SU-8 surface (Vilares et al. 2010). Afterwards, patterning of the metal layer was performed by using a standard photolithography process and different selective wet chemical etchings.

In order to define the electrodes and connecting pads while insulating the metal tracks, a thin SU-8 layer $(1 \mu \mathrm{m})$ was processed on top of the wafer. For that, firstly, the wafer was exposed again to low-power oxygen plasma to promote the adhesion between SU-8 layers. Then, SU-8 2005 was spin-coated and baked at $65^{\circ} \mathrm{C}$ for $2 \mathrm{~min}$ and at $95^{\circ} \mathrm{C}$ for 10 min. The minimum thickness of this layer only required a UV-exposure with a dose of 80 $\mathrm{mJ} / \mathrm{cm}^{2}$ followed by a post-exposure baking made at $65^{\circ} \mathrm{C}$ for 1 min and ramped up to $95^{\circ} \mathrm{C}$ where the wafer was baked for $3 \mathrm{~min}$. After being cooled down the wafer was developed in a PGMEA-based bath for $45 \mathrm{~s}$, rinsed and dried as it had been done before. Again a hard bake at $120^{\circ} \mathrm{C}$ for $20 \mathrm{~min}$ in a hotplate was also performed.

Finally, the whole wafer was immersed in a HF-based bath to etch the $\mathrm{SiO}_{2}$ sacrificial layer, releasing the SU-8 flexible sensing devices. To facilitate the use of the corneal sensing devices, they were directly connected to a printed circuit board (PCB) by means of zero insertion force (ZIF) connectors. For that, the connecting pads of the sensing device were designed to match the specifications of a 10-channel ZIF connector. With this ready-to-use connector, no further encapsulation process was needed, resulting in valuable time and cost-savings.

\section{Electrode modification with black platinum}

As it is well-known, the use of the tetrapolar method minimizes the parasitic effects produced by the electrode-electrolyte interface impedance. However, problems when measuring using high impedance electrodes appear due to the voltage drop produced by the polarization current of the differential amplifier needed to reject the generated current-carrying electrodes. To avoid this issue, a reduction of the electrode impedance is strictly neecessary. For this, among the different methods described in the literature (Gabriel et al. 2008), electrochemically coating of the electrodes with a porous layer of black platinum (Robinson, 1968; Gabriel et al., 2007) has been chosen because of its versatility, quickness and easy implementation in our laboratory (Guimerà et al. 2012). Therefore, a customized process to simultaneously platinize the ten gold electrodes of individual devices was carried out by a 3 min electroplating in a platinum chloride solution (hydrochloric acid $0.1 \mathrm{M}, 2.3 \%$ platinum (IV) chloride and $0.023 \%$ lead (IV) acetate $99 \%$ ) at $200 \mu \mathrm{A}$. The result of this coating is shown in Fig. 2a, where a bare gold electrode is shown together with an electrochemically coated with black platinum electrode.

\section{Impedance sensor characterization}

In order to characterize the electrode-electrolyte interface impedance, a home-made impedance analysis system (Calderón et al. 2009; Guimera et al. 2008) was used. For that, the sensing device was immersed in a physiological saline solution $(0.9 \%$ wt. $\mathrm{NaCl}$, resistivity at $298 \mathrm{~K}=71.3 \Omega \mathrm{cm}$ ) where the electrode impedance was measured versus a platinum reference electrode (Radiometer Analytical) within the $100 \mathrm{~Hz}$ to $100 \mathrm{kHz}$ frequency range. Moreover, these measurements were repeated before and after each series of in vivo experiments to verify the stability of the electrodes. Finally, and using a home-made four-electrode impedance analysis system (Guimerà et al. 2009) and the same physiological saline solution, tetrapolar impedance measurements (within the $100 \mathrm{~Hz}$ to $1 \mathrm{MHz}$ frequency range) were performed to determine the useful frequency band. 

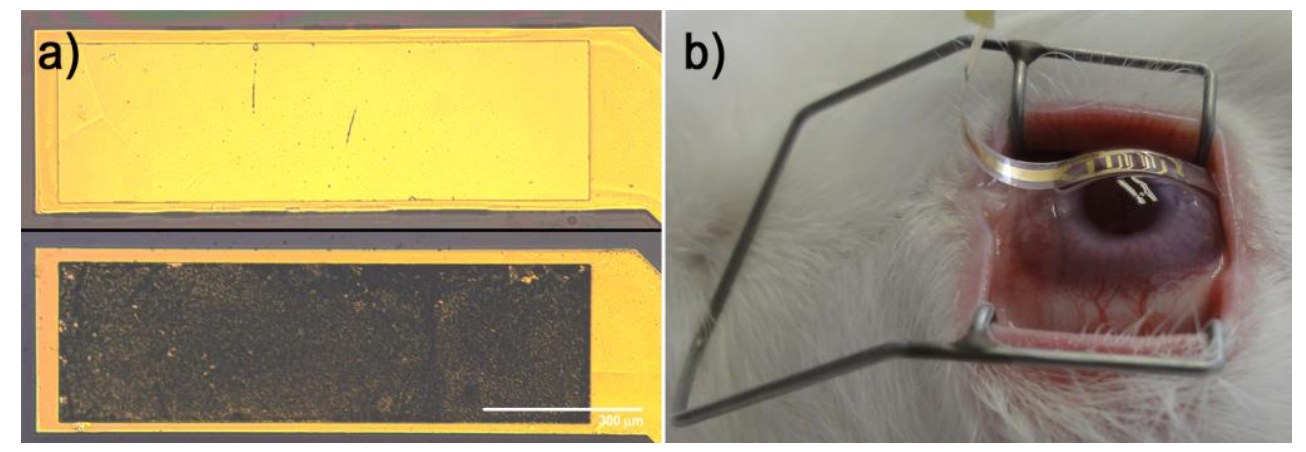

Fig. 2 (a) Optical microscope image of a bare gold electrode just after the fabrication process (up) and after being electrochemically coated with black platinum. (b) Image of how the sensing device is applied to the rabbit's eye during the in vivo experiments

\section{Experimental procedures}

The ability of the proposed sensor to asses the corneal epithelium permeability was evaluated by increasing the permeability of rabbit corneal epithelium via topical instillation of $\mathrm{BAC}$ at different concentrations. To verify the obtained results, the impedance measurements, performed as it is shown in Fig. 2b, were correlated with the corneal epithelium permeability to sodium fluorescein. In particular, corneas used for both characterization techniques were different but equally treated. Moreover, all experimental procedures were conducted under the supervision of the University of Valladolid ethics committee, conformed to the EU guidelines for handling and care of laboratory animals and following the guidelines of the ARVO Statement for the Use of Animals in Ophthalmic and Vision Research.

\section{Increase of corneal epithelium permeability by topical BAC application}

A total of 50 New Zealand white rabbits weighing between 2.5 and $3 \mathrm{~kg}$ were used for these experiments. The rabbits were anaesthetized with a single intramuscular injection of $50 \mathrm{mg} / \mathrm{kg}$ of ketamine (Imalgene $1000 \AA$, Merial) plus $7 \mathrm{mg} / \mathrm{kg}$ of Xilacine (Rompun ${ }^{\circledR}$, Bayer). In each studied animal, one eye was used as control while the damage was induced in the other one (treated eye). Thus, both the control (S) and treated $\left(\mathrm{B}_{\mathrm{x}}\right)$ group were composed of 50 eyes. Moreover, and to avoid the influence of postural alterations, the treated eye was chosen alternately. The damage induced in the treated eyes was accomplished by applying one drop (approximately $50 \mu \mathrm{l}$ ) of BAC solution every minute for 5 minutes up to a total of 5 drops.

Additionally, the treated group was divided in 5 subgroups composed by 10 eyes. For each subgroup a different $\mathrm{BAC}$ solution concentration was used $\left(\mathrm{B}_{1}\right.$ : $\mathrm{BAC}$ at $0.01 \%, \mathrm{~B}_{2}$ : $\mathrm{BAC}$ at $0.02 \%, \mathrm{~B}_{3}: \mathrm{BAC}$ at $0.05 \%, \mathrm{~B}_{4}: \mathrm{BAC}$ at $0.1 \%$ and $\mathrm{B}_{5}: \mathrm{BAC}$ at $\left.0.2 \%\right)$. Furthermore, in the control group an innocuous $\mathrm{NaCl} 0.9 \%$ wt. solution was applied using the same protocol.

\section{Impedance measurements.}

A home-made tetrapolar impedance analysis system (Guimerà et al. 2009) was used to perform impedance spectroscopy measurements as it was described in a previous work (Guimera et al. 2012). This system was complemented with a home-made multiplexer allowing the acquisition of the impedance measurement of the 4 different electrode configurations in an automatic fashion. With the whole system, it takes approximately 1 minute to acquire 20 frequency points within the $100 \mathrm{~Hz}$ to $1 \mathrm{MHz}$ frequency range. The measurement device complies with IEC60601-1 electrical safety and electromagnetic compatibility requirements. Impedance measurements were carried out at three different times; the first one was performed before the treatment $(\mathrm{C})$ in order to measure the basal 
value, the second one just after the BAC solution instillation $\left(\mathrm{S}, \mathrm{B}_{\mathrm{x}}\right)$, and the last one 15 minutes after the application of the last drop $\left(\mathrm{PB}_{\mathrm{x}}\right)$.

\section{Epithelial permeability to sodium fluorescein measurements}

Corneal epithelial permeability to fluorescein was evaluated as an indicator of corneal epithelial integrity. In brief, $50 \mu 1(1 \mathrm{mg} / \mathrm{ml}$ ) of sodium fluorescein (Sigma, 46960) were applied to the ocular surface 15 minutes after BAC instillation. Ten minutes after fluorescein administration, animals were euthanized with intravenous pentobarbital at 200 $\mathrm{mg} / \mathrm{kg}$ (Sigma, P3761) and corneas were excised. Homogenization of each cornea was performed in $300 \mu \mathrm{l}$ of PBS using an Ultra-turrax mixer (IKA) and samples were centrifuged at $14000 \mathrm{rpm}$ and $4^{\circ} \mathrm{C}$ during 10 minutes. Fluorescence in supernatants and in a fluorescein standard was measured using a Victor II plate reader (Perkin Elmer) by duplicate. The concentration of fluorescein was expressed in mg per cornea.

\section{Results and discussion}

\section{Impedance sensor characterization}

As it has been mentioned in the previous section, electrode-electrolyte interface impedance measurements have been performed to characterize the electrode after being electrochemically modified with black platinum. In particular, these results at different stages of the electrode modification are shown in Fig. 3a. There, it can be seen that, as expected, the deposition of a black platinum layer decreases the interface impedance values (70-fold at $100 \mathrm{~Hz}$ ) due to the increase of the electrode roughness and, thus, its effective area. It is also worthy to note that the same measurements performed after each series of in vivo experiments presented only minor changes, proving the stability of the applied electrode modification process (impedance increase at $100 \mathrm{~Hz}<25 \%$, data not shown).
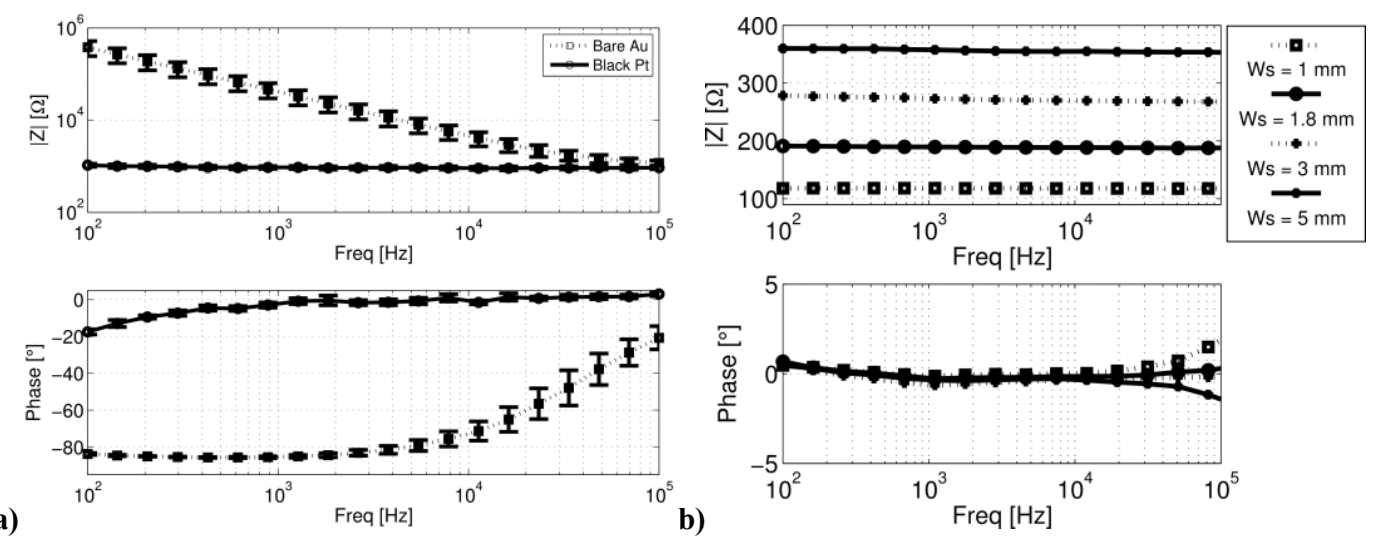

Fig. 3 Results of the impedance sensor characterization: (a) electrode-electrolyte impedance modulus and phase shift of sensor electrodes measured in $\mathrm{NaCl}$ wt. $0.9 \%$ at different stages of the electrode modification: bare gold electrode (dot line) and after black platinum deposition (solid line). Results are expressed as mean \pm standard deviation $(n=20)$. (b) Measured impedance modulus and phase shift of a $\mathrm{NaCl}$ wt. $0.9 \%$ solution using the final impedance sensor modified with black platinum (four-electrode measurement) for the four different sensor configurations. Results are expressed as mean \pm standard deviation $(\mathrm{n}=5)$

Additionally, Fig. $3 \mathrm{~b}$ shows the results of the tetrapolar measurements for a $\mathrm{NaCl} 0.9$ wt.\% solution using the four different geometric configurations of the impedance sensing device. Ideally, in the analyzed frequency range, the measured impedance of a saline solution using the tetrapolar method should be characterized by a flat modulus and a phase shift of $0^{\circ}$ for the whole spectrum (Grimnes and Martinsen 2008). However, this expected behavior was not accomplished at frequencies greater than $300 \mathrm{kHz}$. This can be 
attributable to the capacitive coupling of the wires, which is strongly manifested in the measurements; therefore, the useful frequency band ranges from $100 \mathrm{~Hz}$ to $300 \mathrm{kHz}$.

\section{Epithelial injury produced by topical BAC application}

Experimental results obtained after topical instillation of different BAC solutions are shown in Fig. 4 and 5 (for a better interpretation of these results, label captions of each group are summarized in Table 1). In particular, Fig. $4 \mathrm{a}$ and $4 \mathrm{~b}$ show the full impedance spectrum in both the Bode and Nyquist representation. These results are presented as mean of all the performed measurements for each group (only the data obtained with the $5 \mathrm{~mm}$ wide configuration is shown). As expected, a decrease in both the impedance module and phase, which is inversely proportional to the applied BAC concentration, is clearly observed.
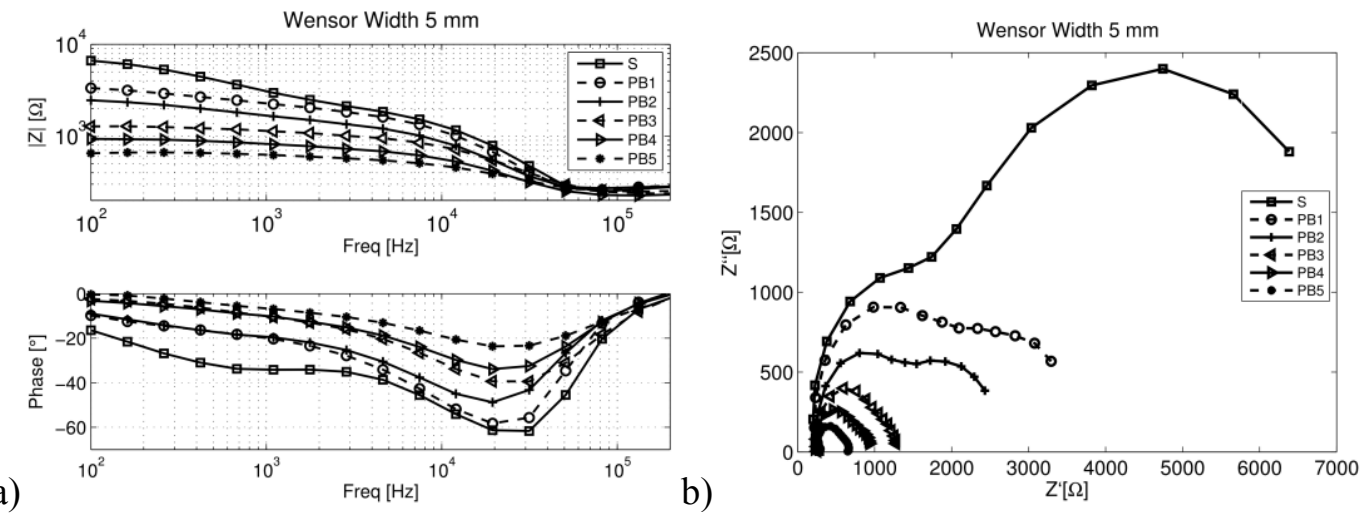

Sensor Width $5 \mathrm{~mm}$

c)

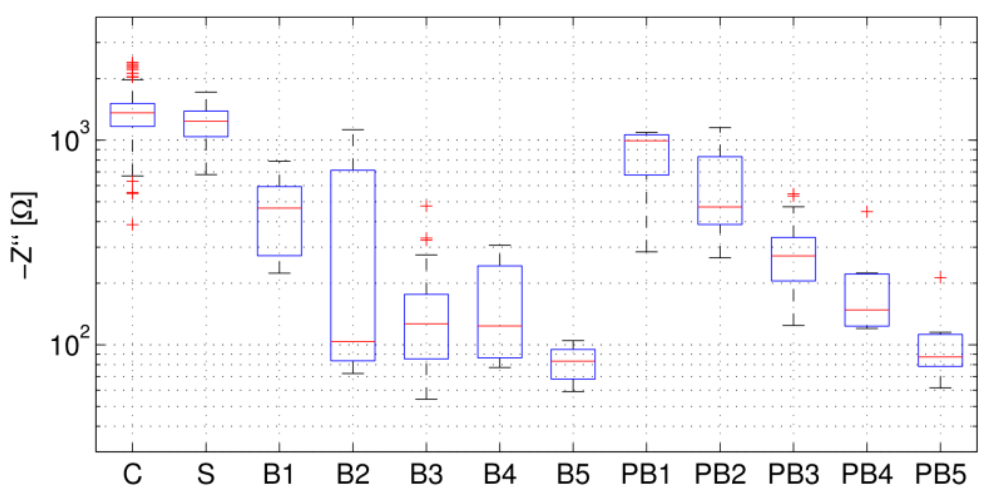

Fig. 4 Experimental impedance measurements for the eyes treated with different BAC dilutions (C: pre-treatment, $\mathrm{S}$ : saline solution, $\mathrm{B}_{1}$ : $\mathrm{BAC}$ at $0,01 \%, \mathrm{~B}_{2}$ : $\mathrm{BAC}$ at $0,02 \%, \mathrm{~B}_{3}$ : $\mathrm{BAC}$ at $0.05 \%$, $\mathrm{B}_{4}$ : $\mathrm{BAC}$ at $0.1 \%$ and $\mathrm{B}_{5}$ : BAC at $0.2 \%$ ). (a) Bode and (b) Nyquist representation of the mean values for each group, respectively. (c) Value of the imaginary part of the measured impedance at $2 \mathrm{kHz}$; the Boxplot representation shows the mean value, the dispersion of the measurements grouped by quartiles (boxes and bars) and the outlier values (crosses) for each group. $\mathrm{B}_{\mathrm{x}}$ groups correspond to the measurements performed just after the treatment and the $\mathrm{PB}_{\mathrm{x}}$ groups to the same measurements performed 15 minutes after the injury

The imaginary part of the measured impedance at $2 \mathrm{kHz}$ was chosen as a prospective indicator of the epithelium permeability. This choice is based on the performed FEM simulations (Fig. 1), which shows that this frequency range is the most sensitive to changes in the passive electrical properties of the epithelium layer. The experimental results of this indicator are shown in Fig. $4 \mathrm{c}$ for all groups using a Boxplot representation. As expected, the impedance measured before treatment (C) and the impedance measured in the control group (S), which was treated with innocuous saline solution, are in the same value range without significant statistical difference. Consequently, this range can be considered as the normality and can be associated to a healthy epithelium. For the rest of the groups, it is interesting to note that the values for the measurements performed 15 
minutes after the injury $\left(\mathrm{PB}_{\mathrm{x}}\right)$ are slightly higher than the measurements performed immediately after injury $\left(\mathrm{B}_{\mathrm{x}}\right)$. Moreover, their dispersion is drastically reduced, especially when the BAC concentration is lower than $0.05 \%$. This fact can be attributed to a stabilization of the damage produced by the injury as it has been reported in a previous work (Kusano et al. 2010), where the impact of the BAC in the permeability and passive electrical properties could be observed just few seconds after its application. Moreover, it has been also reported that the tear produces a dilution of the applied BAC reducing the effects of the injury (Chetoni et al. 2003) which also contributes to the stabilization of the induced damage. Accordingly, and due to its stability and repeatability, the measurements performed 15 minutes after injury will be chosen in the succeeding analysis.

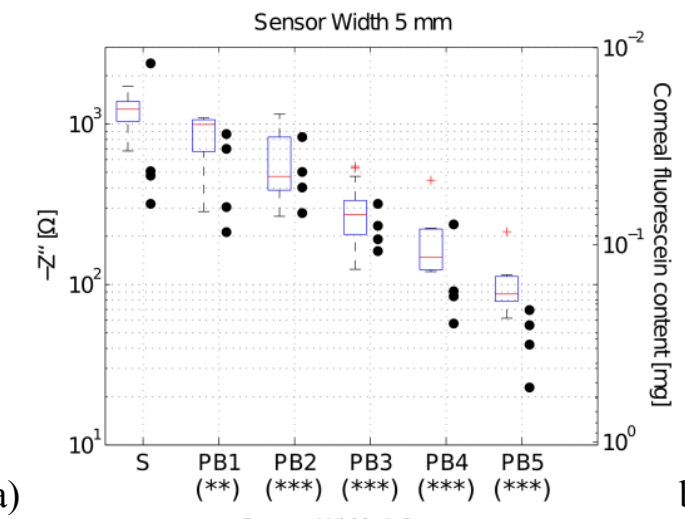

a)

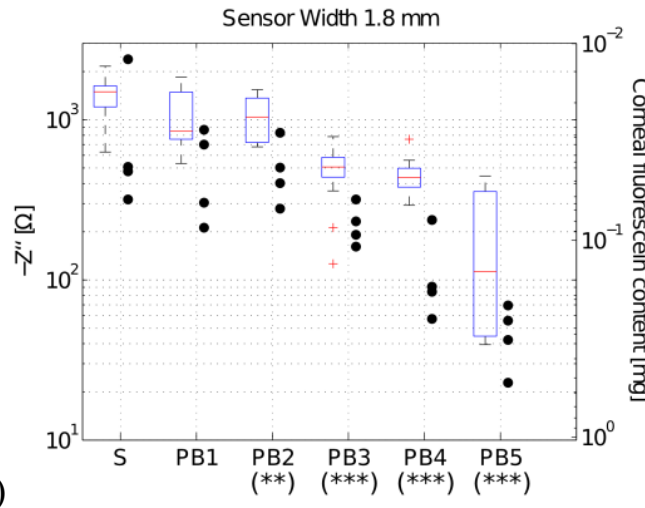

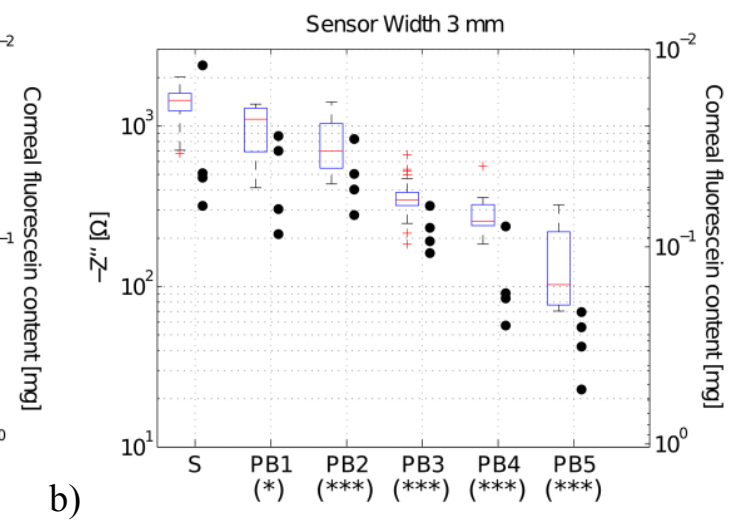

b)

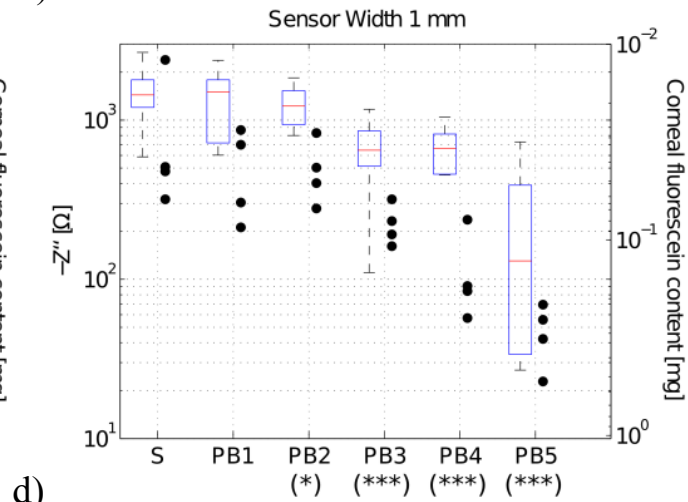

Fig. 5 Experimental results for the eyes treated with different $\mathrm{BAC}$ dilutions $\left(\mathrm{S}, \mathrm{PB}_{\mathrm{x}}\right)$ where the left $\mathrm{Y}$-axis represents the imaginary part of the measured impedance at $2 \mathrm{kHz}$. These results are presented in a Boxplot which shows the mean value, the dispersion of the measurements grouped by quartiles (boxes and bars) and the outlier values (crosses). The right $\mathrm{Y}$-axis represents the permeability to sodium fluorescein measurements of the corneal epithelium with the obtained values presented as points. The different graphs correspond to the results obtained using the different electrode configurations: (a) $\mathrm{W}_{\mathrm{s}}=5 \mathrm{~mm}$, (b) $\mathrm{W}_{\mathrm{s}}=3 \mathrm{~mm}$, (c) $\mathrm{W}_{\mathrm{s}}=1.8 \mathrm{~mm}$ and (d) $\mathrm{W}_{\mathrm{s}}=1$ mm. $\left({ }^{* * *} \mathrm{p}<0.001 ;{ }^{* *} \mathrm{p}<0.01 ;{ }^{*} \mathrm{p}<0.1\right.$ with unpaired student's t-test)

Fig. 5 shows the values of the proposed indicator used to evaluate the epithelium permeability for all the different electrode configurations. This data were compared with the results of the epithelial permeability to sodium fluorescein measurements, which are showed in the secondary axis. Concerning the fluorescein uptake measurements (same data plotted in all graphs of Fig. 5 and showed in Table 1) it can be observed that the results correlate directly with the applied BAC concentration; a correlation that has been previously reported by other authors (Chen et al. 2011). In particular, it was observed a 2 fold increase in the case of PB3 group (BAC at $0.05 \%$ ), a 4 fold increase the case of PB4 group (BAC at $0.1 \%$ ) and an 8 fold increase in the case of PB5 group (BAC at $0.2 \%$ ). In addition, for lower BAC concentrations a slight difference between control and PB1 and PB2 groups was also observed ( $0.01 \%$ and $0.02 \%$ respectively). To summarize, it can be concluded that the applied experimental procedure produces a progressive increase in the 
epithelial permeability and thus, it can be considered suitable for the evaluation of the proposed sensing device.

Focusing on the data from the impedance measurements, a progressive decrease in their values is observed as the BAC concentration increases. In order to evaluate which sensor configuration has a better sensitivity, unpaired student's t-test has been performed by comparing all groups with the control group ( $<<0.01$ was considered statistically significant). From this study, it can be concluded that in all the sensor configurations there is a significant difference between the groups corresponding to higher BAC concentrations (groups PB3, PB4 and PB5). However, for the lower BAC concentration groups (PB1 and $\mathrm{PB} 2$ ), only the $5 \mathrm{~mm}$ wide sensor is able to differentiate between them (e.g. control and PB1 group -BAC at $0.01 \%$ - can be differentiate within a significance level of $\mathrm{p}<0.01$ ). These results are in accordance with the FEM simulations presented in Fig. $1 \mathrm{~b}$, where the $5 \mathrm{~mm}$ wide configuration presents a major contribution of the epithelium layer and minor contribution of the tear film. Thus, it can be concluded that the $5 \mathrm{~mm}$ configuration is the most appropriate to use in order to detect any alteration in the epithelium permeability.

Furthermore, the results obtained in this work can be compared with the results that have been already published by different authors. In particular, the reported values of in vivo TER measurements reported by Kusano et al. and Chen et al. (Chen et al. 2012; Kusano et al. 2010) for low BAC concentrations (up to $0.02 \%$ ) show similar trends as the one obtained with the non-invasive impedance measurement method that has been described in this work. A significant decrease in the impedance values is observed in all cases, although the different measuring protocols and methods employed make it complex to compare the exact values of each work (Chetoni et al. 2003). Moreover, in a work from Uematsu et al. (Uematsu et al. 2007) a decrease of around the $80 \%$ of the initial value in the case of BAC concentration of $0.05 \%$ is reported. This result is in accordance with the value obtained for this specific BAC concentration using the noninvasive method described in this paper and showed in Table 1.

Table 1. Summary of the experimental data for the topical instillation of different BAC solutions and for the corneal epithelium wound-healing process. Data presented as mean \pm Std from 4 eyes per group in the case of corneal fluorescein content, 10 eyes per BAC treated group and 50 eyes per $\mathrm{NaCl}$ group in the case of impedance measurements

\begin{tabular}{ccccccc} 
& & & \multicolumn{2}{c}{$\begin{array}{c}\text { Corneal fluorescein } \\
\text { content }[\mathrm{mg}]\end{array}$} & $\begin{array}{c}\text { Imaginary part of } \\
\text { impedance at } 2 \mathrm{kHz} \\
{[\mathrm{k} \Omega]}\end{array}$ \\
\cline { 4 - 7 } $\begin{array}{c}\text { Group } \\
\text { caption }\end{array}$ & Treatment & Concetration & Mean \pm Std & $\Delta(\%)$ & Mean \pm Std & $\Delta(\%)$ \\
\hline $\mathrm{S}$ & $\mathrm{NaCl}$ & $0.9 \%$ & $0.04 \pm 0.02$ & $0 \%$ & $1.22 \pm 0.23$ & $0 \%$ \\
& & & & & & \\
$\mathrm{~PB}_{1}$ & & $0.0125 \%$ & $0.05 \pm 0.02$ & $32.5 \%$ & $0.85 \pm 0.28$ & $-30.3 \%$ \\
$\mathrm{~PB}_{2}$ & & $0.025 \%$ & $0.04 \pm 0.01$ & $20.0 \%$ & $0.58 \pm 0.29$ & $-52.5 \%$ \\
$\mathrm{~PB}_{3}$ & $\mathrm{BAC}$ & $0.05 \%$ & $0.08 \pm 0.02$ & $120.0 \%$ & $0.28 \pm 0.10$ & $-77.0 \%$ \\
$\mathrm{~PB}_{4}$ & & $0.1 \%$ & $0.17 \pm 0.07$ & $327.5 \%$ & $0.19 \pm 0.10$ & $-84.4 \%$ \\
$\mathrm{~PB}_{5}$ & & $0.2 \%$ & $0.33 \pm 0.14$ & $725.0 \%$ & $0.10 \pm 0.04$ & $-91.8 \%$
\end{tabular}

\section{Conclusions}

In previous studies, a method to non-invasively assess corneal epithelial permeability through tetrapolar impedance measurements was presented and experimentally validated. Here, a SU-8 based sensing device is presented. The flexibility of this novel sensing 
device dramatically improves the application method, ensuring the electric contact between the electrodes and the corneal surface without applying pressure. Furthermore, the influence of the tear film in the measurements can be reduced because of the increased separation between the electrodes. With the obtained results it can be stated that with the presented sensing device and using the previously reported tetrapolar impedance method, it is possible to discern between lower and time-dependent alterations in the corneal epithelium permeability after instillation of very low concentrated BAC solution $(0.01 \%)$. Moreover, the obtained results are comparable with previously reported in-vivo TER measurements performed by invasive electrodes. Therefore, we conclude that the flexible sensing device presented here can be used as a non-invasive tool for assessing the corneal epithelial layer function. This new approach can be used for in vivo studies or in clinical practice, filling the gap existing for corneal function evaluation methods. Moreover, the improvements presented in this work may be applied to detect alterations in the endothelium permeability in a non-invasive way.

\section{Acknowledgments}

We thank to Antoni Ivorra for his contribution in the FEM modeling and sensor conception. This work has been funded by the projects SAF2009-14724-C02-02 from the Spanish Ministry of Science and Innovation, IPT-2012-0438-010000 and SAF2012-40227-C02-02 from Spanish Ministry of Economy and Competitiveness and the European Regional Development Fund.

\section{Conflict of interest}

Laboratorios SALVAT S.A. holds the patent WO2011/107645 A1

\section{References}

Ayaki M, Iwasawa A (2011) Cell Viability of Four Corneoconjunctival Cell Lines Exposed to Five Preservatives and a Surfactant Used for Infection Control in Eyedrops. Biocontrol Science 16:117-121.

Baudouin C, Labbé A, Liang H, et al. (2010) Preservatives in eyedrops: The good, the bad and the ugly. Progress in Retinal and Eye Research 29:312-334. doi: 10.1016/j.preteyeres.2010.03.001

Calderón E, Melero A, Guimerà A (2009) Portable Device for Microelectrode Array Bioimpedance Measurements. In: Dössel O, Schlegel WC (eds) World Congress on Medical Physics and Biomedical Engineering, September 7 - 12, 2009, Munich, Germany. Springer, pp 883-886

Cavet ME, VanDerMeid KR, Harrington KL, et al. (2010) Effect of a novel multipurpose contact lens solution on human corneal epithelial barrier function. Contact Lens and Anterior Eye 33:S18-S23.

Chen W, Hu J, Zhang Z, et al. (2012) Localization and Expression of Zonula Occludins-1 in the Rabbit Corneal Epithelium following Exposure to Benzalkonium Chloride. PLoS ONE 7:e40893. doi: 10.1371/journal.pone.0040893

Chen W, Li Z, Hu J, et al. (2011) Corneal Alternations Induced by Topical Application of Benzalkonium Chloride in Rabbit. PLoS One. doi: 10.1371/journal.pone.0026103

Chetoni P, Burgalassi S, Monti D, Saettone MF (2003) Ocular toxicity of some corneal penetration enhancers evaluated by electrophysiology measurements on isolated rabbit corneas. Toxicol In Vitro 17:497-504.

Furrer P, Mayer JM, Gurny R (2002) Ocular tolerance of preservatives and alternatives. European Journal of Pharmaceutics and Biopharmaceutics 53:263-280. 
Gabriel G, Gómez-Martínez R, Villa R (2008) Single-walled carbon nanotubes deposited on surface electrodes to improve interface impedance. Physiol Meas 29:S203S212. doi: $10.1088 / 0967-3334 / 29 / 6 /$ S18

Geselowitz DB (1971) An Application of Electrocardiographic Lead Theory to Impedance Plethysmography. IEEE Trans Biomed Eng BME-18:38-41. doi: 10.1109/TBME.1971.4502787

Grimnes S, Martinsen ØG (2007) Sources of error in tetrapolar impedance measurements on biomaterials and other ionic conductors. J Phys D: Appl Phys 40:9-14. doi: $10.1088 / 0022-3727 / 40 / 1 / \mathrm{S} 02$

Grimnes S, Martinsen ØG (2008) Bioimpedance and bioelectricity basics. Academic Press

Guimera A, Calderon E, Los P, Christie AM (2008) Method and device for bioimpedance measurement with hard-tissue applications. Physiol Meas 29:S279S290.

Guimerà A, Gabriel G, Parramon D, et al. (2009) Portable 4 Wire Bioimpedance Meter with Bluetooth Link. In: Dössel O, Schlegel WC (eds) World Congress on Medical Physics and Biomedical Engineering, September 7 - 12, 2009, Munich, Germany. Springer, pp 868-871

Guimera A, Gabriel G, Plata-Cordero M, et al. (2012) A non-invasive method for an in vivo assessment of corneal epithelium permeability through tetrapolar impedance measurements. Biosensors and Bioelectronics 31:55-61. doi: 10.1016/j.bios.2011.09.039

Guimera A, Ivorra A, Gabriel G, Villa R (2010) Non-invasive assessment of corneal endothelial permeability by means of electrical impedance measurements. Med Eng Phys 32:1107-1115. doi: 10.1016/j.medengphy.2010.07.016

Keller S, Blagoi G, Lillemose M, et al. (2008) Processing of thin SU-8 films. Journal of Micromechanics and Microengineering 18:

Kimura K, Teranishi S, Fukuda K, et al. (2008) Delayed Disruption of Barrier Function in Cultured Human Corneal Epithelial Cells Induced by Tumor Necrosis Factor- $\alpha$ in a Manner Dependent on NF-KB. IOVS 49:565-571. doi: 10.1167/iovs.07-0419

Kimura K, Teranishi S, Kawamoto K, Nishida T (2011) Protective effect of dexamethasone against hypoxia-induced disruption of barrier function in human corneal epithelial cells. Experimental Eye Research 92:388-393. doi: 10.1016/j.exer.2011.02.013

Kinoshita S, Adachi W, Sotozono C, et al. (2001) Characteristics of the human ocular surface epithelium. Progress in Retinal and Eye Research 20:639-673.

Kusano M, Uematsu M, Kumagami T, et al. (2010) Evaluation of acute corneal barrier change induced by topically applied preservatives using corneal transepithelial electric resistance in vivo. Cornea 29:80-85. doi:

10.1097/ICO.0b013e3181a3c3e6

Lehmann DM, Cavet ME, Richardson ME (2010) Nonclinical safety evaluation of boric acid and a novel borate-buffered contact lens multi-purpose solution, Biotrue ${ }^{\mathrm{TM}}$ multi-purpose solution. Contact Lens and Anterior Eye 33:S24-S32.

Lorenz H, Despont M, Fahrni N, et al. (1997) SU-8: A low-cost negative resist for MEMS. Journal of Micromechanics and Microengineering 7:121-124.

Ma L, Kuang K, Smith RW, et al. (2007) Modulation of tight junction properties relevant to fluid transport across rabbit corneal endothelium. Exp Eye Res 84:790-798. doi: 10.1016/j.exer.2006.12.018

McNamara NA, Fusaro RE, Brand RJ, et al. (1997) Measurement of corneal epithelial permeability to fluoresce in: A repeatability study. Investigative Ophthalmology and Visual Science 38:1830-1839.

Paiva CSD, Corrales RM, Villarreal AL, et al. (2006) Apical Corneal Barrier Disruption in Experimental Murine Dry Eye Is Abrogated by Methylprednisolone and Doxycycline. IOVS 47:2847-2856. doi: 10.1167/iovs.05-1281 
Rojanasakul Y, Robinson JR (1990) Electrophysiological and ultrastructural characterization of the cornea during in vitro perfusion. International Journal of Pharmaceutics 63:1-16.

Schulzke J-D, Günzel D, John LJ, Fromm M (2012) Perspectives on tight junction research. Annals of the New York Academy of Sciences 1257:1-19. doi: 10.1111/j.1749-6632.2012.06485.x

Schwan HP, Ferris CD (1968) Four-Electrode Null Techniques for Impedance Measurement with High Resolution. Rev Sci Instrum 39:481-485. doi: $10.1063 / 1.1683413$

Swamynathan S, Kenchegowda D, Piatigorsky J, Swamynathan S (2011) Regulation of Corneal Epithelial Barrier Function by Krüppel-like Transcription Factor 4. IOVS 52:1762-1769. doi: 10.1167/iovs.10-6134

Swan KC (1944) Reactivity of the ocular tissues to wetting agents. Am J Ophthalmol 27:1118-1122.

Uematsu M, Kumagami T, Kusano M, et al. (2007) Acute Corneal Epithelial Change after Instillation of Benzalkonium Chloride Evaluated Using a Newly Developed in vivo Corneal Transepithelial Electric Resistance Measurement Method. Ophthalmic Res 39:308-314. doi: 10.1159/000109986

Vilares R, Hunter C, Ugarte I, et al. (2010) Fabrication and testing of a SU-8 thermal flow sensor. Sensors and Actuators B: Chemical 147:411-417. doi: 10.1016/j.snb.2010.03.054

Yang F, Patterson RP (2008) A Simulation Study on the Effect of Thoracic Conductivity Inhomogeneities on Sensitivity Distributions. Ann Biomed Eng 36:762-768. doi: 10.1007/s10439-008-9469-0 